\title{
THÉORIE POSTCOLONIALE ET LE SPECTRE DE MARX: À PROPOS DU MARXISME DE VIVEK CHIBBER
}

Par Kolja LINDNER

On ne peut que se féliciter de la récente traduction française de l'important livre Postcolonial Theory and the Specter of Capital de Vivek Chibber ${ }^{1}$. D'une part, il s'agit sans doute de l'une des plus vigoureuses critiques des études postcoloniales dont nous disposons actuellement on y reviendra. D'autre part, l'intérêt de cette publication réside dans la réception particulière de la pensée postcoloniale en France. Celle-ci s'est en effet opérée tardivement, il y a une dizaine d'années, et sur le fond particulier d'un débat sur l'histoire coloniale et la condition postcoloniale dans l'Hexagone ${ }^{2}$. Le malaise qu'éprouve habituellement le monde académique français envers la production intellectuelle anglophone explique ainsi que ce débat ressemble partiellement à une nouvelle entreprise de réinvention de la roue. Mais cette difficulté est aussi la cause d'une première vague de critiques aux relents ethnocentriques visant à défendre l'inventivité francophone en la matière, et à rejeter ce qui est perçu comme une culturalisation postcolonialiste. On peut également ajouter le fait que cette critique est souvent mal fondée sur le plan théorique, ignorant les débats dans la théorie sociale anglo-saxone à propos du couple structure/action, de la notion d'agency ou des représentations sociales ${ }^{3}$.

\section{L’ « IDÉOLOGIE » SUBALTERNISTE EN QUESTION}

L'articulation d'une démarche empirique et théorique qui caractérise l'effort de Chibber lui permet de se détacher de telles polémiques. L'auteur s'efforce ainsi «d'analyser le cadre conceptuel produit par les études postcoloniales pour l'analyse historique et plus précisément pour l'analyse de ce qu'on appelait alors le Tiers-Monde ${ }^{4}$ ». L' « Histoire classique $»$ de la modernisation colonialo-capitaliste des pays du Sud, mobilisée par les études postcoloniales, se voit ainsi récusée. Celle-ci est perçue

comme un phénomène global, bien que son expansion ait pris des formes différentes selon l'époque et le lieu. Même si le monde colonisé y est venu plus tard, il ne faisait guère de doute qu'il suivrait les sillons creusés par le monde avancé. Le moteur de ce processus était constitué par l'industrialisme et les pratiques économiques modernes, conjointement avec les transformations politiques et culturelles. [...] L'Europe offrait au monde en développement une image approximative de son propre avenir ${ }^{6}$.

\footnotetext{
${ }^{1}$ Chibber Vivek, Théorie postcoloniale et le spectre du Capital, trad. fr. Christine Vivier, Toulouse, Éditions de l'Asymétrie, 2017. Nous remercions l'éditeur de nous avoir donné préalablement accès à cette traduction. Sa publication n'ayant pas encore été suffisamment avancée au moment de la rédaction du présent article, nous ne pouvons renvoyer qu'aux chapitres et sous-chapitres du livre de Chibber, mais non pas à des pages précises.

${ }^{2}$ Cf. notamment Bancel Nicolas, Blanchard Pascal et Lemaire Sandrine (dir.), La Fracture coloniale. La société française au prisme de l'héritage colonial, Paris, La Découverte, 2005, et Bancel Nicolas et Blanchard Pascal (dir.), Ruptures postcoloniales. Les nouveaux visages de la société française, Paris, La Découverte, 2010. Sur la « non-réception des Subaltern Studies en France », voir Zancarini-Fournel Michelle, "À propos du 'retard' de la réception en France des Subaltern Studies », Actuel Marx, n 51, 2012, pp. 150-164.

${ }^{3}$ Les livres de Jean-Loup Amselle (L'Occident décroché. Enquête sur les postcolonialismes, Paris, Éditions Stock, 2008) et de Jean-François Bayart (Les études postcoloniales, un carnaval académique, Paris, Karthala, 2010) en témoignent chacun de leur manière.

${ }^{4}$ Chibber Vivek, Théorie postcoloniale et le spectre du Capital, op. cit., ch. 1.2.

${ }^{5}$ Ibidem, ch. 1.3.

${ }^{6}$ Ibidem, ch. 1.3 .
} 
C'est à partir de cette vision de l'histoire identifiée comme un « métarécit marxiste ${ }^{7}$ », que les Subaltern Studies construisent un contre-récit, réhabilitant la "différence historique » du développement indien. Celui-ci serait justement caractérisé par l'échec de la «tendance

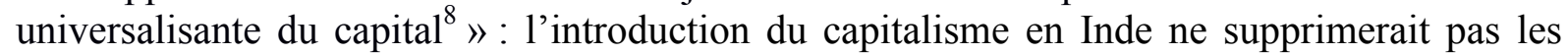
formes de domination précapitalistes. Plus encore, la bourgeoisie indienne (contrairement à celle en occident) s'avérerait incapable de construire un mode de domination de classe hégémonique, c'est-à-dire de présenter ses intérêts comme identiques de ceux des classes dépendantes ${ }^{9}$ et de développer des institutions prônant l'égalité, la liberté, le sécularisme, etc. Selon Chibber, cette approche présente deux implications. La première est que « l'analyse des hiérarchies sociales en Inde ne peut pas s'appuyer sur les catégories de l'économie politique qui repose sur le capital comme mécanisme d'explication ${ }^{10} »$. La critique marxienne de l'économie politique se voit ainsi rangée dans l'inventaire eurocentrique inapte à saisir le développement des pays du Sud : «Un capitalisme spécifiquement postcolonial demande des catégories spécifiquement postcoloniales. ${ }^{11} »$ Ces catégories sont celles du « fragment », de la psychologie, voire de la religion. L'auteur leur reproche ce qu'il identifie comme étant la deuxième conséquence de la vision subalterniste, à savoir de présenter des effets théoricopolitiques qui sont à l'opposé de la visée initiale du projet de l'historiographie indienne : un «hyper avant-gardisme» contrastant grandement avec l'«appel programmatique à reconnaître et respecter l'agency subalterne ${ }^{12} »$, et un « essentialisme culturel ${ }^{13}$ » déplaçant les intérêts matériels dans le domaine d'une psychologie particulière ${ }^{14}$. Au final, «les Subaltern Studies ne peuvent constituer ni une théorie du capitalisme dans sa forme mondialisée, ni sa critique, puisqu'elles proposent systématiquement une interprétation erronée du fonctionnement du capitalisme ${ }^{15} »$.

\footnotetext{
${ }^{7}$ Chakrabarty Dipesh, Provincialiser l'Europe. La pensée postcoloniale et la différence historique, Paris, Éditions Amsterdam, 2009, p. 51.

${ }^{8}$ Chibber Vivek, Théorie postcoloniale et le spectre du Capital, op. cit., titre $5^{\text {ème }}$ chapitre.

${ }^{9} \mathrm{C}$ 'est la fameuse thèse de la « domination sans hégémonie » de Ranajit Guha (Dominance without Hegemony. History and Power in Colonial India, Cambridge Mass./Londres, Harvard University Press, 1997). Il est toutefois symptomatique du caractère réductionniste de la réception de Marx et du marxisme dans les études postcoloniales que l'hégémonie soit identifiée à une simple capacité de «s'exprimer au nom de la nation" divisée en classes sociales (Guha Ranajit, «Quelques questions concernant l'historiographie de l'Inde coloniale », Tracés. Revue de Sciences humaines, $\mathrm{n}^{\circ}$ 30, 2016, p. 198). Le concept central d'Antonio Gramsci dispose cependant d'une plus grande complexité. On peut notamment distinguer deux volets, l'un stratégique (formation d'alliances, composées d'éléments hétérogènes ou bien de forces diverses) et l'autre motivationnel (plausibilité mentale d'un ordre dominant, donc non seulement de son acceptation passive, mais aussi de sa réception productive, de sa mise en pratique et de son développement créatif par les dominé.e.s). Je développe ces deux aspects dans Lindner Kolja, "'Muddled thinking' : Stuart Hall et la théorie d'hégémonie » in Malek Bouyahia, Franck Freitas et Karima Ramdani (dir.), L'Optimisme de la volonté. Stuart Hall, une sentinelle critique pour une hégémonie de gauche, Paris, La Dispute, 2017 (à paraître) ainsi que dans Lindner Kolja, Die Hegemoniekämpfe in Frankreich. Laizismus, politische Repräsentation und Sarkozysmus, Hambourg, Argument, 2017, pp. 26-66.

${ }^{10}$ Chibber Vivek, Théorie postcoloniale et le spectre du Capital, op. cit., ch. 5.1.

${ }^{11}$ Ibidem, ch. 5.1 .

${ }^{12}$ Ibidem, ch. 7.4 .

${ }^{13}$ Ibidem, ch. 8.4.

${ }^{14}$ Sumit Sarkar, faisant d'abord lui-même partie des subalternistes, est probablement le précurseur le plus direct de la critique chibbérienne: voir notamment «Le déclin du subalterne dans les Subaltern Studies», Contretemps, $\mathrm{n}^{\circ}$ 20, 2007, pp. 37-49. Pour une critique de l'orientation religieuse du dernier Dipesh Chakrabarty, voir Sarkar Sumit, «Subalternité et histoire globale. Entretien de Déborah Cohen et Urs Lindner avec Sumit Sarkar», Actuel Marx, $\mathrm{n}^{\circ}$ 50, 2011, pp. 207-217. Chibber soutient cependant que cette critique de l'approche subalterniste a surtout été formulée au début et au milieu des années 1990 «avant que certains de ses arguments clés n'aient été complètement développés ou aient même vu le jour » (Chibber Vivek, Théorie postcoloniale et le spectre du Capital, op. cit., ch. 1.4) et que nous arrivons donc « à un moment où le réexamen du projet semble indispensable, maintenant que les membres du collectif ont eu le temps d'en expliciter plus en détail les implications » (ibidem, ch. 1.4).

${ }^{15}$ Chibber Vivek, Théorie postcoloniale et le spectre du Capital, op. cit., ch. 11.1.
} 
À l'encontre de $l^{\prime}$ " idéologi $\mathrm{i}^{16}$ » des subalternistes, Théorie postcoloniale et le spectre du Capital se veut donc une réhabilitation du marxisme. Selon Chibber, celui-ci dispose d'un meilleur outillage pour mener à bien une analyse des pays du Sud. L'argument central porte ainsi sur les formes du capitalisme dans ces aires, voire sur la nature du capitalisme en général. Et face à la révélation impitoyable des failles théoriques du discours postcolonial, on n'a peu de mal à suivre l'auteur dans son analyse du déploiement du capitalisme en Inde. Ce qui pose cependant davantage problème, c'est la conception de marxisme même qui soutient cette entreprise, celui-ci étant présenté comme une machine théorique omnipotente. Une telle conception est non seulement imaginaire, mais elle bloque de surcroît toute compréhension du point départ des études postcoloniales. Celles-ci ont d'abord été, Chibber le reconnaît explicitement, " une innovation au sein de la théorie marxiste, non une rupture radicale avec celle-ci ${ }^{17} \gg$. Ainsi, comme nous le verrons tout au long de cet article, les critiques qu'avancent les subalternistes ne sont pas tirées de nulle part. Elles disposent bel et bien d'un ancrage tout à fait réel dans le discours marxiste.

Sans mettre en question le bien-fondé de la critique chibbérienne, nous devons donc travailler à une conception plus juste du champ théorique marxien et rectifier les défaillances du marxisme, - une entreprise dont l'enjeu théorique et politique dépasse largement le débat à propos de ses avantages et inconvénients par rapport aux études postcoloniales ${ }^{18}$. Dans ce qui suit, nous mettrons en lumière cette difficulté à partir de quatre points ressortant de l'étude de Chibber: l'histoire de la genèse du capitalisme, le travail abstrait, le travail « doublement libre » et la conception des intérêts.

\section{LA GENĖSE DU CAPITALISME}

L' «Histoire classique» que Chibber récuse à juste titre, c'est-à-dire la conception d'un développement schématique et téléologique des sociétés précapitalistes vers celles où « règne le mode de production capitaliste ${ }^{19}{ }^{\prime}$, est bel et bien présente dans l'œuvre marxienne. On peut penser notamment aux articles parus en 1853 dans le New York Daily Tribune, et dans lesquels Marx soutient une vision techno-fonctionnaliste de la modernisation occidentale à travers la soumission coloniale, fortement inspirée de la fantasmagorie européenne d'une société stagnante en Inde et de la philosophie d'histoire hégélienne ${ }^{20}$. Un autre exemple d'une telle conception presque identique à la reformulation de l' «Histoire classique » par Chibber se trouve dans la préface du Livre I du Capital où nous lisons : "Le pays le plus développé industriellement ne fait que montrer à ceux qui le suivent sur l'échelle industrielle l'image de leur propre avenir. ${ }^{21} \gg$ Certes, cette préface s'adresse en particulier au lectorat allemand qui, dans les années 1860, aurait pu prendre le capitalisme pour quelque chose de spécifiquement anglais. En outre, l'adaptation du Livre I du Capital pour la traduction française relativise cette vision : l'expropriation des cultivateur.e.s « ne s'est encore accomplie d'une manière radicale qu'en Angleterre : ce pays jouera donc nécessairement le premier rôle dans

\footnotetext{
${ }^{16}$ Ibidem, titre $11^{\text {ème }}$ chapitre.

${ }^{17}$ Ibidem, ch. 1.2 .

${ }^{18}$ Pour une vue d'ensemble d'autres efforts récents dans le débat à propos du rapport entre marxisme et études postcoloniales, voir Lindner Kolja, «Eurocentrisme, postcolonialisme et marxisme : nouveaux regards ?», Raisons politiques. Revue de théorie politique, ${ }^{\circ}$ 63, 2016, pp. 161-177.

${ }^{19}$ Marx Karl, Le Capital (Paris 1872-1875), Marx-Engels-Gesamtausgabe, section II, tome 7, p. 19.

${ }^{20}$ Voir notamment Marx Karl, « La domination britannique aux Indes », « La compagnie des Indes orientales. Son histoire et ses résultats » et «Les conséquences futures de la domination britannique en Inde », in Karl Marx, Euvres IV. Politique I, Paris, Gallimard, 1994, pp. 714-736. Ces textes montrent des fortes traces notamment de Bernier François, Voyage dans les États du Grand Mogol, Paris, Fayard, 1981 et de Hegel G.W.F., Vorlesungen über die Philosophie der Geschichte, Euvres, tome 12, Francfort s.1.M., Suhrkamp, 1985 , p. 174-218. Marx cite même explicitement, comme inspirant son analyse de la société indienne, la première de ces sources dans une lettre à Engels du 2 juin 1853 (Marx Karl, Engels Friedrich, Correspondance, tome 3, Paris, Éditions Sociales, 1972, p. 378).

${ }^{21}$ Marx Karl, Le Capital, op. cit., p. 12.
} 
notre esquisse [historique de ce processus]. Mais tous les autres pays de l'Europe occidentale parcourent le même mouvement, bien que selon le milieu il change de couleur locale, ou se resserre dans un cercle plus étroit, ou présente un caractère moins fortement prononcé, ou suive un ordre de succession différent ${ }^{22} \gg$. Enfin, dans sa correspondance avec la révolutionnaire russe Vera Zassoulitch en 1881, Marx met non seulement entre guillemets la "'fatalité historique' » de l'expropriation des cultivateur.e.s dans la genèse de la production capitaliste, mais il la voit aussi «expressément restreinte aux pays de l'Europe occidentale $^{23} »$.

On doit cependant retenir que Marx n'a pas été sur une ligne critique de l'eurocentrisme dès le début de sa recherche à propos de la genèse du capitalisme. Il a effectué un cheminement qui fait que l'on peut trouver des textes contradictoires au sein d'une même œuvre ${ }^{24}$. Ces textes ont inspiré différentes approches marxistes de la question, mais il est sans doute exagéré d'affirmer comme le fait Chibber que l'histoire du marxisme au $\mathrm{XX}^{\mathrm{e}}$ siècle serait l'histoire d'une « seule entreprise - comprendre la spécificité de l'Orient ${ }^{25}$ ».

Ce qui est avancé ici par Chibber comme «la » position marxiste est en réalité un courant, certes fort et innovant, mais particulier d'historiographie. Or, ce courant a dû se battre contre l'orthodoxie et le dogmatisme marxiste et est toujours loin d'être hégémonique. Il s'agit du « marxisme politique » de Robert Brenner et d'Ellen M. Wood ${ }^{26}$, qui cherche à réhabiliter la contingence de la genèse du capitalisme, une perspective bien résumée par le sociologue Gerhard Hauck :

La production de marchandises, la propriété privée et le travail salarié, la liberté juridique, l'exploitation des forces de travail fondée sur la contrainte économique (absence de moyens de production), la sûreté du droit et la relative abstinence économique de l'État (largement responsable en même temps de la distinction proprement capitaliste entre économie et politique), l'existence de forces intermédiaires et la séparation du pouvoir religieux et politique, le pillage des régions périphériques et les phases d'essor de la science et de la technique : tout cela constitue - à l'encontre de toutes les positions euro-théorético-modernisantes - des phénomènes que la plupart des sociétés ont vécu à un moment donné de leur histoire. Ces phénomènes ont eu des effets conjoints dans l'Angleterre des $\mathrm{XVII}^{\mathrm{e}}$ et $\mathrm{XVIII}^{\mathrm{e}}$ siècles, ce qui a rendu possible la naissance du capitalisme à un moment singulier de l'histoire ${ }^{27}$.

\footnotetext{
${ }^{22}$ Ibidem, p. 634 .

${ }^{23}$ Marx Karl, «Marx an Zasulič », Marx-Engels-Archiv. Zeitschrift des Marx-Engels-Instituts in Moskau, tome 1, Francfort s.1.M., Marx-Engels-Archiv Verlagsgesellschaft, 1926, p. 341.

${ }^{24} \mathrm{Je}$ ne rentre pas dans la polémique à propos de la «maturation » de Marx, important dans le débat marxiste à la suite la périodisation de l'œuvre marxienne soutenue par Louis Althusser (Pour Marx, Paris, La Découverte, 2005, pp. 23-30). Il n'est pas nécessaire d'être d'accord avec cet auteur pour constater une évolution importante du regard marxien sur les pays du Sud au cours de son œuvre : voir Lindner Kolja, "L'eurocentrisme de Marx : pour un dialogue du débat marxien avec les études postcoloniales », Actuel Marx, $\mathrm{n}^{\circ}$ 48, 2010, pp. 106-128 et, dans une perspective explicitement anti-althussérienne, Anderson Kevin, Marx aux antipodes. Nations, ethnicités et sociétés non occidentales, Paris, Éditions Syllepse, 2015.

${ }^{25}$ Chibber Vivek, Théorie postcoloniale et le spectre du Capital, op. cit., ch. 11.3.

${ }^{26}$ Aston T.H., Philpin C.H.E. (dir.), The Brenner Debate : Agrarian Class Structure and Economic Development in Pre-Industrial Europe, Cambridge, Cambridge University Press, 1985 ; Brenner Robert, Merchants and Revolution: Commercial Change, Political Conflit and London's Overseas Traders 1550-1653, Princeton, Princeton University Press, 1993, et Wood Ellen M., The Origin of Capitalism : A Longer View, Londres/New York, Verso, 2002. Pour une synthèse en langue française, voir également Wood Ellen M., «Le marxisme politique et ses débats. Entretien de Frédérick-Guillaume Dufour et Jonathan Martineau avec Elle Meiksins Wood », Actuel Marx, n 50, 2011, pp. 98-118.

27 Hauck Gerhard, Die Gesellschaftstheorie und ihr Anderes. Wider den Eurozentrismus der Sozialwissenschaften, Münster, Westfälisches Dampfboot, 2003, p. 134 (nous traduisons).
} 
Les révolutions anglaises et françaises ne peuvent donc être perçues comme l'œuvre d'une classe capitaliste montante contre une noblesse féodale réfractaire, mais comme les résultats chacun particulier selon son contexte - d'une lutte populaire qui s'est imposée sans que la bourgeoisie souscriva à une large coalition de forces sociales pour renverser l'ancien régime comme le soutiennent les Subaltern Studies. "S'il y a eu une contribution directe faite par ces deux soulèvements, elle a concerné le renforcement de l'État et non celui du capitalisme ou de la démocratie ${ }^{28}$ », écrit ainsi Chibber. Et cette conclusion a des conséquences sur une figure théorique chère aux subalternistes : «En résumé, la domination sans hégémonie comme Guha la définit - n'est pas une aberration associée au monde postcolonial ou le signe d'un échec d'une révolution bourgeoise. Ce fut et c'est la figure normale du pouvoir bourgeois. $^{29}$ ")

Il faut cependant noter que ce ne sont pas les Subaltern Studies qui ont inventé le modèle théorique qu'elles récusent. Celui-ci provient d'une partie du discours marxiste même, en l'occurrence du «marxisme néo-smithien » que Brenner soumet à une critique fondamentale pour son model commercial de la genèse du capitalisme ${ }^{30}$.

\section{LE TRAVAIL ABSTRAIT}

Un autre exemple d'ambiguïté, portant cette fois davantage sur les écrits de Marx que sur le marxisme, réside dans la définition du travail abstrait qu'ils soutiennent. Rappelons qu'il s'agit du concept avec lequel, dans Le Capital, Marx saisit la commensurabilité des différents travaux « concrets ». Ainsi nous lisons dans le premier chapitre du Livre I :

En fin de compte, toute activité productive, abstraction faite de son caractère utile, est une dépense de force humaine. La confection des vêtements et le tissage, malgré leur différence, sont tous deux une dépense productive du cerveau, des muscles, des nerfs, de la main de l'homme, et en ce sens du travail humain au même titre. La force humaine de travail dont le mouvement ne fait que changer de forme dans les diverses activités productives, doit assurément être plus ou moins développée pour pouvoir être dépensée sous telle ou telle forme. Mais la valeur des marchandises représente purement et simplement le travail de l'homme, une dépense de force humaine en général $^{31}$.

Dans l'histoire du marxisme, cette définition a fait débat. On a interrogé en fait ce qu'il y avait de spécifiquement capitaliste dans une telle «dépense, dans le sens physiologique, de force humaine ${ }^{32} »$. Ainsi, l'économiste russe Isaak Roubine, plus tard victime du stalinisme, soutenait dans les années 1920 que l'échange est ici saisi par « un concept physiologique, dépouillé de tout élément social et historique ${ }^{33} »$. Roubine doit ainsi être considéré comme un précurseur de l'interprétation selon laquelle la critique de l'économie politique effectue une révolution scientifique partielle par rapport au champ théorique d'auteurs comme Adam Smith et David Ricardo. Ainsi Marx romprait avec leur anthropologie, individualisme, anhistorisme et empirisme, tout en reproduisant ces défauts à diverses occasions ${ }^{34}$. En l'occurrence, parler de « dépense productive du cerveau, des muscles, des nerfs, de la main de l'homme », et d' "une dépense de force humaine en général », fait preuve d'une telle

\footnotetext{
${ }^{28}$ Chibber Vivek, Théorie postcoloniale et le spectre du Capital, op. cit., ch. 3.3.

${ }^{29}$ Ibidem, ch. 4.4 .

${ }^{30}$ Brenner Robert, «The Origins of Capitalist Development : A Critique of Neo-Smithian Marxism », New Left Review, ${ }^{\circ} 104,1977$, pp. 25-92.

${ }^{31}$ Marx Karl, Le Capital, op. cit., p. 27.

${ }^{32}$ Ibidem, p. 29.

${ }^{33}$ Roubine Isaak I., Essais sur la théorie de la valeur de Marx, Paris, François Maspero, 1977, p. 181.

${ }^{34}$ Cf. Heinrich Michael, Die Wissenschaft vom Wert. Die Marxsche Kritik der politischen Ökonomie zwischen wissenschaftlicher Revolution und klassischer Tradition, Münster, Westfälisches Dampfboot, 1999.
} 
conception anthropologique et empiriste du travail abstrait. Cependant Marx va dans un sens tout à fait différent à d'autres moments de l'argumentation. Le travail abstrait représenterait une abstraction qui n'aurait rien à voir avec l'activité tangible car «c'est l'échange seul qui opère cette réduction ${ }^{35}$ " des travaux concrets. Dans le remaniement de la première édition allemande du Capital, cette idée est exprimée encore plus clairement : "La réduction des différents travaux concrets à cet abstrait du travail humain égal s'opère seulement à travers l'échange qui de fait met les produits de différents travaux sur un pied d'égalité. ${ }^{36}$ "

Le concept du travail abstrait joue un rôle important dans le rejet subalterniste de la critique de l'économie politique comme eurocentrique, ainsi que dans la revendication de catégories spécifiquement postcoloniales pour saisir le capitalisme des pays du Sud. On doit apprécier l'intégralité de l'argument de Chakrabarty qui écrit sous l'influence de son collègue du département d'histoire de l'université de Chicago, Moishe Postone ${ }^{37}$. Pour l'historien indien le concept de travail abstrait «permet à Marx d'expliquer comment le mode de production capitaliste est parvenu à extraire de peuples et d'histoires différents une unité commune et homogène permettant de mesurer l'activité humaine ${ }^{38} »$. Selon Chakrabarty, cette abstraction ne se réalise qu'en pratique.

Notons l'expression : «l'abstraction [...] devient pratiquement vraie ». Marx n'aurait pu l'indiquer plus clairement: le travail abstrait n'est ni une substance, ni du travail physiologique, ni une somme calculable d'énergie musculaire et nerveuse. Ce terme renvoie à une pratique, à une activité, à la mise en œuvre concrète de travail d'abstraction, en quoi il est très semblable aux stratégies analytiques de l'économie, lorsqu'il y est question de cette catégorie abstraite nommée « travail » ${ }^{39}$.

L'interprétation que Chakrabarty propose s'inscrit donc pleinement dans l'ambiguité marxienne, ce qui est d'autant plus difficile à saisir qu'il rejette, en référence aux lectures de Rubine et de Postone $^{40}$, la conception physiologique du travail abstrait, pour s'en tenir fermement à une conception empiriste. Et il en conclut que le travail abstrait serait une discipline imposée aux travailleur.e.s pour rendre leur activité concrète conforme à des standards de commensurabilité, le travail concret apparaissant ainsi comme « une trace derridienne de quelque chose qui ne peut pas être enfermée, un élément qui défie constamment le capital et la marchandise de l'intérieur ${ }^{41} \gg$.

Chibber a donc grandement raison de réfuter de telles interprétations, mais une défense honnête de Marx reconnaîtrait les ambivalences du concept de travail abstrait dans la critique de l'économie politique même, et conclurait à la nécessité d'efforts théoriques pour les lever. La seule ambivalence de Marx reconnue par Chibber est cependant celle qui demeurerait dans le fait « qu'il n'a jamais exposé méthodiquement de thèse sur les identités sociales dans le

\footnotetext{
${ }^{35}$ Marx Karl, Le Capital, op. cit., p. 55.

${ }^{36}$ Marx Karl, Das Kapital. Kritik der politischen Ökonomie. Erster Band (Hamburg 1872), Marx-EngelsGesamtausgabe, section II, tome 6, p. 41 (nous traduisons).

37 Postone a proposé une interprétation du Capital qui l'hégélianise davantage en soutenant une priorité du conceptuel et un absolutisme de l'abstrait: «la généralité abstraite historiquement constituée par le travail abstrait établit aussi 'le travail concret' et 'la valeur d'usage' en tant que catégories générales » (Postone Moishe, Temps, travail et domination sociale. Une réinterprétation de la théorie critique de Marx, Paris, Éditions Mille et une nuits, 2009, p. 228). Nous y lisons également que les rapports sociaux capitalistes auraient « un caractère formel et objectif particulier, ils ne sont pas ouvertement sociaux et se caractérisent par la dualité antinomique totalisante du concret et de l'abstrait, du particulier et de l'homogénement général » (ibidem, p. 230).

${ }^{38}$ Chakrabarty Dipesh, Provincialiser l'Europe, op. cit., p. 100.

${ }^{39}$ Ibidem, p. 105.

${ }^{40}$ Ibidem, p. 104.

${ }^{41}$ Chakrabarty Dipesh, "Marx After Marxism : History, Subalternity, and Difference », in Makdisi Saree, Casarino Cesare, et Karl Rebecca E. (dir.), Marxism Beyond Marxism, Londres/New York, Routledge, 1996, p. 60 (nous traduisons).
} 
capitalisme $^{42} »$. Le défaut de Marx résiderait donc dans ce qu'il n'aurait pas effectué et non pas dans ce qu'il a fait.

\section{LE TRAVAIL « DOUBLEMENT LIBRE »}

Chibber soutient que le concept de travail abstrait « est parfaitement en mesure d'appréhender la diversité sociale $^{43}$ » et, de ce fait, entièrement compatible avec les hiérarchies sociales précapitalistes qui peuvent avoir une forte influence dans les pays du Sud. Cette thèse illustre un autre problème qui émerge d'un manque d'honnêteté intellectuelle par rapport au cadre théorique marxien. Il s'agit du travail « doublement libre ». Dans Le Capital, Marx stipule qu'afin de pouvoir accumuler, les capitalistes doivent trouver sur le marché

le travailleur libre, et libre à un double point de vue. Premièrement le travailleur doit être une personne libre, disposant à son gré de sa force de travail comme de sa marchandise à lui ; secondement, il doit n'avoir pas d'autre marchandise à vendre ; être, pour ainsi dire, libre de tout, complètement dépourvu des choses nécessaires à la réalisation de sa puissance travailleuse ${ }^{44}$.

De nombreuses recherches ont démontré que cette présupposition est fausse, et ceci vaut non seulement pour le capitalisme dans les pays du Sud. Le travail libre n'a même pas été la forme généralisée du travail dans le capitalisme occidental, ni au moment de sa genèse, ni au moment de sa consolidation. Ainsi, l'historien Peer Vries démontre «à quel point le travail non-libre a été important dans et pour la Grande-Bretagne, ce 'berceau du capitalisme' et la première puissance industrielle mondiale ${ }^{45} »$. Et sur ce point, le pays le plus développé industriellement ne faisait que montrer l'image des réalités mondiales. Ainsi, on estime qu'à la fin du XVIII ${ }^{\mathrm{e}}$ siècle seulement $4 \%$ du travail a été libre, et qu' « il y a des bonnes raison d'affirmer que l'importance du travail non-libre a augmenté plutôt que diminué au début de l'ère moderne ${ }^{46} »$.

La première période de l'industrialisation en Grande-Bretagne n'a pas été caractérisée par une prédominance grandissante du travail libre. La Grande-Bretagne ne s'est pas industrialisée plus tôt que le reste de l'Europe occidentale à cause du « travail libre ». C'est plutôt le contraire qui semble être vrai [...]. Les travailleurs d'usine, l'incarnation par excellence de la Révolution Industrielle et du nouveau «travail libre », n'ont constitué qu'une très petite partie de toute la population travailleuse en Grande-Bretagne. De plus, une très grande partie des travailleurs salariés des usines britanniques avant et au début de l'industrialisation ont été des femmes et des enfants ${ }^{47}$.

À l'échelle globale, Marx a certes été conscient de l'enjeu économique et politique de la

\footnotetext{
${ }^{42}$ Chibber Vivek, Théorie postcoloniale et le spectre du Capital, op. cit., ch. 6.2.

${ }^{43}$ Ibidem, ch. 6, intro.

${ }^{44}$ Marx Karl, Le Capital, op. cit., p. 137.

${ }^{45}$ Vries Peer, State, Economy and the Great Divergence. Great Britain and China, 1680s-1850s, Londres/New York, Bloomsbury, 2015, p. 333 (nous traduisons).

${ }^{46}$ Idem.

${ }^{47}$ Ibidem, p. 335. L'historien Arno J. Mayer soutient même qu'au XIX ${ }^{\mathrm{e}}$ et au début du XX ${ }^{\mathrm{e}}$ siècle, cet « arriéré » ne se réduit pas au seul niveau économique. Au contraire, il caractériserait aussi les domaines politiques, sociaux et culturels : " les éléments 'prémodernes' n'ont pas été des restes déclinants et fragiles d'un passé en train de disparaître, mais plutôt l'essence même de l'ordre social et politique régnant en Europe » (Mayer Arno J., The Persistence of the Old Regime. Europe to the Great War, New York, Pantheon Books, 1981, p. 5 et suiv. ; nous traduisons).
} 
forme la plus extrême du travail non-libre, à savoir l'esclavage ${ }^{48}$. Mais il ne l'a pas intégré dans sa conception critique de l'économie capitaliste. Dans ce cadre théorique, la mise en esclavage ferait partie des "procédés idylliques d'accumulation primitive qui signalent l'ère capitaliste à son aurore ${ }^{49} »$, mais non pas du capitalisme développé. Ici, les esclavagistes ne sont que des capitalistes parce qu'ils « existent en tant qu'anomalies à l'intérieur d'un marché mondial reposant sur le travail libre ${ }^{50} »$.

Cette conception découle en partie de la perspective particulière qu'adopte l'auteur du Capital, comme l'a justement précisé l'historien Michael Zeuske: «après l'échec des révolutions de 1848-1849, Marx a prévu, réalisé et écrit une histoire théorique du capital ainsi que du développement du capitalisme industriel avec la production en masse, et non pas une histoire théorique du travail (qui aurait dû être plus large, plus bigarrée et plus globale). ${ }^{51}$ » Ainsi, différent.e.s auteur.e.s ont conclu «que Marx n'a pas justifié de manière cohérente le privilège qu'il accorde au travail salarié productif dans sa théorie de la valeur ${ }^{52} »$. Par conséquent, ces critiques ont revendiqué l'élargissement de la théorisation marxienne de façon à ce que «le travail productif des esclaves et d'autres travailleurs non-libres soit reconnu comme partie intégrante de l'économie capitaliste ${ }^{53}$ ». Dans le même sens, la philosophe Nancy Fraser a soutenu que le capitalisme ne reposait pas seulement sur l'exploitation, mais aussi sur l'expropriation dont les résultats sont mis en valeur, c'est-à-dire intégrés dans le marché « libre ». C'est une des raisons pour lesquelles elle plaide pour « une conception étendue du capitalisme comme un ordre social institutionnalisé, également construit sur l'expropriation. Loin d'être réduit à un seul système économique, la société capitaliste englobe des arrangements extra-économiques qui permettent l'expansion infinie et l'appropriation privée de la plus-value ${ }^{54} »$.

Certes, par rapport à ces différenciations, « la conviction que la production capitaliste peut se dispenser de coercition interpersonnelle dans les relations de classe repose sur des arguments superficiels ${ }^{55} »$, comme le note Chibber. Par ailleurs, il est sûrement plus approprié de concevoir l'universalisation du capital comme un processus qui « se déroule si la stratégie de reproduction des agents évolue vers une dépendance au marché56 $"$, et non pas par une émancipation juridique quelconque des exploité.e.s. Enfin, on ne peut certainement pas identifier l'universalisation du capital à une homogénéisation du travail libre, ni voir dans la persistance des formes de domination «précapitalistes» dans le monde colonial une contradiction à l'exploitation capitaliste. Cette dernière est, au contraire, à attribuer à « la

\footnotetext{
${ }^{48}$ Voir notamment Marx Karl, « Die amerikanische Frage in England », « Der nordamerikanische Bürgerkrieg » et «Der Bürgerkrieg in den Vereinigten Staaten », in Marx Karl, Engels Friedrich, Marx-Engels-Werke, tome 15, Berlin, Dietz, 1980, pp. 304-313 et 329-347.

${ }^{49}$ Marx Karl, Le Capital, op. cit., p. 668. Pour Marx, il s'agit bien entendu de la mise en esclavage des populations racisées. La critique de l'économie politique manque d'une analyse du rôle des femmes dans la transition au capitalisme. Il a fallu des recherches féministes ultérieures pour élucider cette histoire (voir notamment Federici Silvia, Caliban and the Witch: Women, the Body and Primitive Accumulation, New York, Autonomedia, 2004).

${ }^{50}$ Marx Karl, Ökonomische Manuskripte $1857 / 58$ (Grundrisse der Kritik der politischen Ökonomie), MarxEngels-Gesamtausgabe, section II, tome 1.2, p. 415 (nous traduisons).

${ }^{51}$ Zeuske Michael, « Karl Marx, Sklaverei, Formationstheorie, ursprüngliche Akkumulation und Global South. Eine globalhistorische Skizze », in Wemheuer Felix (dir.), Marx und der globale Süden, Cologne, Papy Rossa, 2016, p. 124 (nous traduisons).

${ }^{52}$ Roth Karl Heinz et Van der Linden Marcel, «Ergebnisse und Perspektiven », in Van der Linden Marcel et Roth Karl Heinz (dir.), Über Marx hinaus. Arbeitsgeschichte und Arbeitsbegriff in der Konfrontation mit den globalen Arbeitsverhältnissen des 21. Jahrhunderts, Berlin, Assoziation A, 2009, p. 586 (nous traduisons).

53 Idem.

${ }^{54}$ Fraser Nancy, «Expropriation and Exploitation in Racialized Capitalism : A Reply to Michael Dawson », Critical Historical Studies, vol. 3, $\mathrm{n}^{\circ}$ 1, 2016, p. 173 (nous traduisons).

${ }^{55}$ Chibber Vivek, Théorie postcoloniale et le spectre du Capital, op. cit., ch. 5.3.

${ }^{56}$ Ibidem, ch. 5.2
} 
façon particulière dont le capital a pénétré ces zones ${ }^{57}$ ». Mais, encore une fois, ce ne sont pas les seules études postcoloniales qui soutiennent de tels points de vue. C'est Marx lui-même ! Et il a fallu tout un travail critique d'historien.ne.s et de théoricien.ne.s, dont quelques un.e.s ont été cité.e.s ici, pour sortir des conceptions superficielles du travail libre que le texte de Marx diffuse aussi bien que les études subalternes.

\section{LA CONCEPTION DES INTÉRÊTS}

À l'encontre de l'essentialisme culturel des subalternistes qui explique l'action sociale dans les pays du Sud par une psychologie particulière, Chibber cherche à « rétablir l'idée d'intérêts universels, propre aux Lumières ${ }^{58} »$. Ces intérêts seraient « des facteurs causaux et explicatifs dans l'agency social, quel que puisse être le contexte culturel des agents ${ }^{59} \gg$. De fait, les «besoins objectifs ${ }^{60} »$, notamment le « simple besoin de bien-être physique ${ }^{61}$ », éclairerait «l'essentiel de l'histoire politique ${ }^{62} »$. Cet universalisme des intérêts objectifs complèterait celui de «l'élan universalisant du capital, qui a opéré en Orient aussi bien qu'en Occident, bien qu'à des rythmes différents et de façon inégale ${ }^{63}$ ». Enfin l'universalisme des intérêts résisterait à celui du capital : «s'il existe une véritable source d'opposition à la dynamique d'universalisation du capital, elle se trouve dans la lutte tout aussi universelle des classes subalternes pour défendre leur humanité essentielle. ${ }^{64} »$

Derrière ces thèses se dissimule à nouveau un problème théorique d'ampleur que Chibber semble simplement ignorer. Il s'agit ce celui de la conception « objective » des intérêts que Marx a notamment défendu dans ses œuvre de jeunesse et avec lui une partie du marxisme. Cette idée consiste à dire que sous le régime capitaliste, les orientations normatives et les objectifs d'action des différents groupes sociaux seraient directement et uniquement déterminés par leurs positions sociales respectives. En reprenant cette conception, Chibber reproduit une confusion classique de la tradition marxiste qui tend «à identifier intérêts éthique et positionnel ${ }^{65} \gg$.

Deux conséquences, à l'opposé des visées de Chibber, résultent de cette identification. Premièrement, elle fait tomber l'auteur dans une essentialisation des subalternes. Tandis que ces dernier.e.s sont en réalité différencié.e.s par leurs appartenances de caste et de genre, l'idée de l'universalité de leurs intérêts les transforme en un groupe social homogène. Pourtant, il est « erroné de supposer qu'une position commune dans un rapport de domination mènerait automatiquement à des intérêts convergents d'acteurs respectifs. Car, les acteurs peuvent interpréter un seul et même intérêt positionnel de manière différente. Et la superposition de différentes structures sociales peut aussi générer des intérêts tout à fait nouveaux ${ }^{66}$ ». La conception chibbérienne des intérêts «objectifs" semble ici reproduire deux lacunes d'une bonne partie du marxisme, à savoir le refus d'intégrer la différenciation féministe de l'intersectionnalité et le rejet d'un débat ouvert à propos de questions éthiques.

Deuxièmement, la conception marxienne des intérêts «objectifs » ne semble pas loin de l' «hyper avant-gardisme» des Subaltern Studies que Chibber récuse. Car pour le Marx des années 1840 , c'est uniquement le prolétariat qui possède « un caractère d'universalité par

\footnotetext{
${ }^{57}$ Ibidem, ch. 5.3 .

${ }^{58}$ Ibidem, ch. 8, intro.

${ }^{59}$ Ibidem, ch. 8.4.

${ }^{60}$ Ibidem, ch. 8.4.

${ }^{61}$ Ibidem, ch. 8.4.

${ }^{62}$ Ibidem, ch. 8.4.

${ }^{63}$ Ibidem, ch. 8.6.

${ }^{64}$ Ibidem, ch. 9.4.

${ }^{65}$ Schuck Hartwig, « Wie objektiv sind Interessen ? Facette und Funktionen des Interessenbegriffs in kritischen Analysen sozialer Verhältnisse », Zeitschrift für kritische Sozialtheorie und Philosophie, vol. 1, n 2, 2014 , p. 317 (nous traduisons).

${ }^{66}$ Ibidem, p. 308 et suiv.
} 
l'universalité de ses souffrances ${ }^{67} »$. Dans son émancipation serait donc contenue « l'émancipation humaine générale - cette dernière y étant contenue parce que c'est toute la servitude humaine qui est enveloppée dans le rapport du travailleur à la production, et que tous les rapports de servitude ne sont que des modifications et des conséquences de ce rapport ${ }^{68} »$. Dans la tradition marxiste, c'est cet avant-gardisme qui a constitué un obstacle pour concevoir toute forme d'intersectionnalité, il suffit de penser à la hiérarchisation des rapports de domination que Mao opère avec sa distinction entre « contradiction principale » et «contradiction subordonnée ${ }^{69}$ ». À nouveau, l'enjeu d'une conception adéquate du capitalisme dans les pays du Sud ne consiste pas simplement à jouer le marxisme contre les études postcoloniales, en l'occurrence un avant-gardisme contre un autre, pour s'imaginer dans la caste des théoriquement intouchables. L'enjeu consiste à engager des efforts critiques pour remédier aux failles analytiques, qu'elles proviennent du marxisme ou des études postcoloniales.

\section{CONCLUSION : LES DÉFIS DE L'AUTOCRITIQUE ET DU PLURALISME}

Théorie postcoloniale et le spectre du Capital a suscité de vifs débats à sa parution ${ }^{70}$, mais cette controverse n'a fait que conforter son auteur, en bonne partie en raison de la qualité insuffisante des critiques qui visaient à mettre en cause les propos de Chibber (tandis que les éloges dominaient). Cette situation pourrait produire une illusion de consécration dont les effets risquent d'être terribles. À l'encontre d'un tel confort de pensée, l'avenir de la pensée marxienne semble plutôt résider dans des exercices qui ont posé problème à de larges parties de la tradition marxiste, à savoir sa capacité d'autocritique et d'acceptation du pluralisme.

Contre un dogmatisme se posant en gardien du temple, il semble important de retrouver une capacité d'autocritique et de renouvellement dans le procès de formation et de diffusion d'une théorie critique du capitalisme global. Certes, cet exercice peut paraitre plus facile aujourd'hui, vu que les appareils d'États et les partis cherchant à garantir une certaine «ligne » ont disparu. Mais ils ont trouvé leurs successeurs dans un marxisme académique, certes beaucoup plus marginal que les partis d'autrefois, mais d'autant plus résistant à une vraie discussion critique de Marx que leur propre position institutionnelle semble menacée ${ }^{71}$. C'est certainement une des raisons pour lesquelles l'avenir du débat critique sur Marx est moins dans les mains de ceux et celles qui se revendiquent de manière féroce de son œuvre, que dans celles d'intellectuel.le.s qui considèrent ses écrits comme une contribution cardinale à une théorie critique de la société existante à enrichir par les connaissances produites en théorie féministe, en histoire globale, etc.

Le pluralisme, c'est-à-dire l'art du compromis et de la médiation, apparait intimement lié à la capacité à effectuer son autocritique. Cent ans après la Révolution russe, il est indubitable que les différences d'ordre politique mais aussi théorique (les deux étant souvent liées) «ne peuvent être réglées par l'autorité d'un savoir plus profond ${ }^{72} »$. Et ce défi n'est pas levé par la seule reconnaissance du drame qu'a produit la «solution bureaucratique et autoritaire à ce problème $^{73}$ ». Car les tentations d'un centralisme léniniste pour contrer les critiques soulignant l'intersectionnalité des rapports de domination restent d'une malheureuse

\footnotetext{
${ }^{67}$ Marx Karl, Critique du droit politique hégélien, Paris, Éditions Sociales, 1975, p. 211.

${ }^{68}$ Marx Karl, Manuscrits économico-philosophiques de 1844, Paris, Vrin, 2007, p. 127.

${ }^{69}$ Tsé-toung Mao, À propos de la contradiction, Paris, La Fabrique, 2008, pp. 136-145.

${ }^{70}$ Ce débat est documenté dans Warren Rosie (dir.), The Debate on Postcolonial Theory and the Specter of Capital, Londres/New York, Verso, 2017.

${ }^{71} \mathrm{Ne}$ citons, à titre d'exemple de l'orthodoxie et du dogmatisme contre lesquels porte notre discussion, que l'étude Globalisation and the Critique of Political Economy: New Insights from Marx's Writings (Abingdon/New York, Routledge, 2015) de Lucia Pradella.

${ }^{72}$ Geras Norman, «Classical Marxism and Proletarian Representation », New Left Review, n 125, 1981, p. 83 (notre traduction).

${ }^{73}$ Ibidem, p. 85.
} 
actualité $^{74}$. Marx avait une réponse claire face à de telles scléroses : «ce qu'il y a de certain c'est que moi, je ne suis pas Marxiste ${ }^{75}$. »

\footnotetext{
${ }^{74}$ Voir Callinicos Alex, « Is Leninism finished ? », http://socialistreview.org.uk/376/leninism-finished.

${ }^{75}$ Marx selon les dires d'Engels dans sa lettre à Edouard Bernstein du 2/3 novembre 1882 dans Marx Karl, Engels Friedrich, Marx-Engels-Werke, tome 35, Berlin, Dietz, 1967, p. 388.
} 